\title{
BMJ Open WeChat-based vestibular rehabilitation for patients with chronic vestibular syndrome: protocol for a randomised controlled trial
}

Peixia Wu (D) , ${ }^{1}$ Yafang Wan, ${ }^{1}$ Yu Zhuang, ${ }^{2}$ Chennan Wang, ${ }^{2}$ Shuxin $\mathrm{Xi},{ }^{3}$ Hui Zhu ${ }^{4}$

To cite: Wu P, Wan Y, Zhuang Y, et al. WeChat-based vestibular rehabilitation for patients with chronic vestibular syndrome: protocol for a randomised controlled trial. BMJ Open 2021;11:e042637. doi:10.1136/ bmjopen-2020-042637

- Prepublication history and additional material for this paper are available online. To view these files, please visit the journal online (http://dx.doi. org/10.1136/bmjopen-2020042637).

Received 11 July 2020

Revised 02 February 2021

Accepted 04 February 2021

Check for updates

(C) Author(s) (or their employer(s)) 2021. Re-use permitted under CC BY-NC. No commercial re-use. See rights and permissions. Published by BMJ.

${ }^{1}$ Nursing Department, Eye and ENT Hospital of Fudan University, Shanghai, China ${ }^{2}$ Fudan University School of Nursing, Shanghai, China ${ }^{3}$ Medical Department, Eye and ENT Hospital of Fudan University, Shanghai, China ${ }^{4}$ Shanghai Chaorun Network Technology Ltd, Shanghai, China

\section{Correspondence to}

Dr Shuxin Xi; shuxinxi71@126.com and

Mr Hui Zhu;

zhuorson@163.com

\section{ABSTRACT}

Introduction Dizziness is one of the most common symptoms seen in chronic vestibular syndrome, which has been linked to an increased risk of falls, substantial disability and negative psychological consequences. Recent evidence demonstrated that vestibular rehabilitation therapy (VRT) is effective for treating chronic vestibular symptoms. However, the delivery of VRT remains challenging because of lack of facility, insufficient qualified physiotherapist resources, as well as being in the actual situation of the pandemic. WeChat, the most widely used mobile app in China, offers a more viable way of delivering VRT than traditional office-based approaches do. This study aimed to evaluate the effectiveness of the WeChatVRT programme for patients with chronic vestibular syndrome.

Methods and analysis This is a parallel-group, assessorblinded randomised controlled trial. Fifty patients who experienced chronic vestibular symptoms longer than 3 months will be randomised into either the WeChat-VRT group or the usual care (UC) group. Participants in the WeChat-VRT group will receive 8-week VRT mainly through the WeChat app. Participants in the UC group will receive once-weekly VRT in the clinic for 8 weeks and remaining time home-based exercise. Outcome assessments will take place at baseline and at the 8th, 12th and 24th weeks after randomisation. The primary outcome will be the change from baseline to the eighth week on the patients' functional improvements quantified by the Functional Gait Assessment (FGA). The secondary outcomes will include dynamic balance function, emotional well-being, and vestibular activity and participation level. Intention-to-treat analysis will be performed using generalised estimation equation modelling.

Ethics and dissemination The trial has been reviewed and approved by the Institutional Review Board of Eye and Ear Nose Throat Hospital of Fudan University (reference number 2017047/1). The study findings will be disseminated via peer-reviewed journals and conferences. Trial registration number ChiCTR2000029457; Preresults.

\section{INTRODUCTION}

When a vestibular disorder damages the peripheral vestibular system and the vestibular compensation fails, chronic vestibular
Strengths and limitations of this study

- This study will be the pioneer in identifying the efficacy of social media app as a novel strategy of delivering vestibular rehabilitation therapy (VRT) to patients with chronic vestibular syndrome.

- This study is designed as a parallel-group, assessorblinded randomised controlled trial, which is able to provide a robust answer for the study question.

- The relatively long follow-up period, combined with both subjective and objective outcomes being measured, enabled this study to fully reflect the efficacy of the proposed intervention.

- There is no guarantee that subjects in the WeChatVRT group can perform the exercise correctly since no on-site coaching is being offered.

- The VRT protocol may not be customised enough, even though the VRT is catered to each patient at the initial clinic visiting; thus, modification cannot be ensured throughout the study course.

symptoms can occur, which was prescribed as chronic vestibular syndrome by the Classification Committee of the Barany Society. ${ }^{12}$ Vestibular symptoms are common in otologic clinical practice, the most distinct of which are dizziness and imbalance. Chronic vestibular syndrome, widely defined as symptoms being persistent for $\geq 3$ months, is nonspecific and often goes untreated, which leads to disability and handicap in general population. ${ }^{3}$ In a cross-sectional study, one in five people of working age had symptoms of dizziness in the previous month; half of them were handicapped to some degree and $40 \%$ of them reported occupational difficulties. ${ }^{4}$ In an epidemiological study with more than 15000 participants aged 14 years to older than 75 years, dizziness is reported to prevent $22 \%$ of affected individuals from performing daily activities. ${ }^{5}$ Furthermore, as indicated in Bisdorff et als survey, dizziness, vertigo and unsteadiness were interconnected and occurred mostly in combinations rather than 
in isolation. ${ }^{6}$ Older adults who suffer from dizziness are prone to fall and avoid doing physical activity in order not to provoke symptoms. This avoidance behaviour may result in increasing frailty, reducing balance confidence and less social engagement. Furthermore, dizziness substantially interfered with activities or led to medication intake or medical consultation. ${ }^{7}$

Each peripheral vestibular disorder has a substantial potential to result in chronic vestibular syndrome, commonly including benign paroxysmal positional vertigo (BPPV), Meniere's disease, vestibular migraine (migraine-related dizziness), vestibular neuritis and other conditions. ${ }^{12}$ The chronic symptoms caused by these disorders can be primary or secondary, but is usually characterised by a history of vertigo attack. The exact mechanisms leading to chronic vestibular syndrome in patients with previous vestibular disorder are unknown but there is agreement that the problem is multifactorial. ${ }^{8}$ Review of the management of chronic vestibular syndrome indicated that no medication has well-established evidence nor is any medication suitable for long-term use. ${ }^{9}$

Vestibular rehabilitation therapy (VRT), first described by Cawthorne and Cooksey in the 1940s, is an exercisebased therapy consisting of eye, head and body movements aimed to optimise vestibular compensation and habituation. ${ }^{10}$ VRT approach typically includes a combination of three main elements: (1) gaze stability exercises; (2) habituation exercises, including optokinetic exercises; and (3) balance and gait training in various conditions. ${ }^{10}$ Recently, VRT has been recommended as a first-line treatment for chronic dizziness. ${ }^{11}$

Although evidence indicated that VRT is the most effective treatment for chronic vestibular syndrome, its accessibility is limited in China. There are several reasons for this underutilisation. First, there is a lack of qualified physiotherapist resource and facility. Despite that we have seen a great number of physiotherapists being trained in recent years, most of them are working in neurology department in large tertiary hospitals, typically providing neurological rehabilitation for patients suffering from stroke or trauma brain injury. Very few therapists are working in vestibular rehabilitation field. Also, lack of necessary facilities, such as training ground and dynamic balance equipment, hinders the applicability of VRT for most hospitals. Second, on the patients' end, physical limitations and financial issues prevent them from attending clinic regularly; especially so when the majority of the patients are senior adults. Internet-based approach may be used in situations such as long distance from the site, with transportation difficulty or being in the actual situation of pandemic. WeChat is the most popular mobile app in China, with currently more than 1 billion monthly active users, offering a feasible way to deliver VRT with its potential to overcome many barriers of the office-based approach. One of the obvious advantages is its popularity and ease of use. Given that WeChat has become an essential daily used application for almost all Chinese people, flexible communication and prompt feedback can be realised through this platform. Participants can access and carry out the VRT exercise at their convenience and pace. With the above-mentioned aspects considered, it is reasonable for us to deliver an online VRT therapy through WeChat (WeChat-VRT) in patients with chronic vestibular syndrome.

Although the use of WeChat as a tool to help people with other chronic conditions has been explored and its effectiveness has been proved as well, ${ }^{12-14}$ there is no study, until today, being done in chronic vestibular syndrome population. If this study yields expected effects in improving balance function and emotional well-being among enrolled participants, it will be an innovative strategy for management of chronic vestibular syndrome. Furthermore, this study would offer empirical data of the effectiveness of WeChat-VRT over the office-based VRT and encourage otologists and physiotherapists to adopt this approach as a standard treatment to optimise patient management.

The purpose of this study was to verify the broad range of efficacy of WeChat-VRT for patients with chronic vestibular syndrome. We hypothesise that WeChat-VRT will not be inferior to office-based VRT (usual care) in terms of its efficacy in improving patients' balance function, functional capacity and well-being.

\section{METHODS AND ANALYSIS}

\section{Study design, setting and participants}

This study is designed as a randomised controlled prospective single-blinded study. Since VRT is a physical intervention, no blinding will be possible for either the physiotherapist or the patients. However, the outcomes assessors, a graduate student majored in clinical rehabilitation, working as a salaried research assistant in charge of questionnaire collection, and a technician, who is responsible for performing all balance examinations, will be blinded regarding the group allocation throughout the study.

This study will be conducted in an Eye and Ear Nose Throat (ENT) specialised tertiary care hospital in Shanghai. The hospital serves a population of 23 million persons in Shanghai. It also serves as a referral centre accepting patients from all over the country. The principal investigator $(\mathrm{PW})$ is a licensed physiotherapist with 4 years' experience in treating patients with chronic dizziness. The number of patients with dizziness is about 200 per year in the study setting. It is expected to enrol 50 subjects over a 12-month period.

The eligibility criteria are as follows: (1) a diagnosis of a peripheral vestibular disorder; (2) age of 18-80; (3) an experience of vestibular symptoms and/or unsteadiness longer than 3 months; (4) a local resident with ability to attend clinic for follow-up examinations; (5) ownership of a smartphone and access to the internet; and (6) willingness to participate and compliance with the proposed training programme. Participants will be excluded if they (1) are diagnosed with BPPV or identifiable 
non-labyrinthine cause of dizziness, for instance, central nervous system involvement; (2) have neuromusculoskeletal disease which influences balance control and gait; (3) are suspected to have fluctuating inner ear disorder (migraine fluctuating symptoms, active Ménière's disease and so on); (4) have contraindications of head movements, for instance, a severe cervical disorder; and (5) have been involved in VR exercises before the trial.

\section{Procedure}

The diagnosis tests for vestibular disorders include detailed history taking, caloric testing, pure tone audiometry, vestibular-evoked myogenic potential and video head impulse testing. Suitable patients will initially be identified by ENT specialists, physiotherapists from the hospital where the trial is conducted. They will be given a brief description of the study per se. If the patients indicate willingness, they will subsequently have the study explained to them in person or by phone. Images such as MRI or CT would be ordered, if necessary, to exclude any central system lesion. After providing completed informed consent forms, subjects will be randomised into two treatment arms: group A, referred to as usual care (UC), will receive outpatient VRT combined with home practice; group B (WeChat-VRT) will be offered a series of VRT videos online through WeChat. In addition to VRT, participants in both groups simultaneously receive the standard level of care in the study setting, without restrictions. That means they could use medications to control dizziness as judged by their ENT specialist. Outcomes will be assessed four times, including a baseline and three follow-ups after allocation: a first follow-up at the 8th week, a second follow-up at the 12th week and a third follow-up at 24th week. Figure 1 provides the study flowchart.

\section{Randomisation and blinding}

Participants will be randomly assigned to two groups with 25 persons in each. The randomisation process will be done by an internet-based randomisation tool offered by China clinical trial registration centre, which is freely available at http://www.medresman.org/login.aspx. The

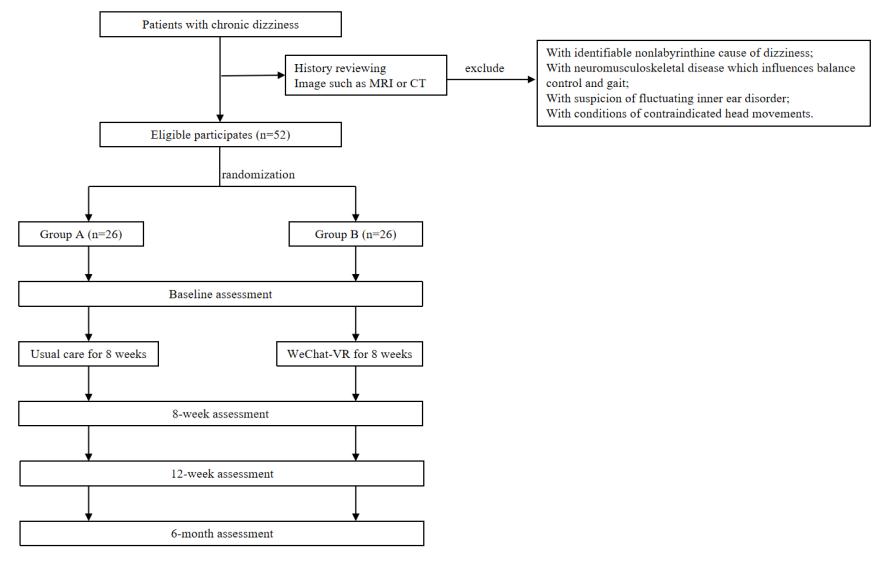

Figure 1 Study flowchart. randomisation sequence will be generated automatically when the participants log into the website and request the allocation number (once an eligible patient is recruited). The randomisation sequences will be kept by an independent statistician and will not otherwise be known to the trial personnel until assignment. Participants will be randomly assigned at a ratio of $1: 1$ and stratified by sex and age ( $\leq 60$ years, $>60$ years) to ensure that these two factors match. As the randomisation is online and automatic, neither the researchers nor the participants can manipulate which treatment the patients will receive; this ensures that group allocations are randomly assigned. The physical therapist will be notified of group allocation after a consent form was obtained and baseline assessments were completed.

\section{Interventions}

The exercise regimen mainly consists of targeted exercise programmes to address functional impairments and to meet individual patient's goal. Targeted exercise involves gaze stabilisation exercises to improve vestibuloocular reflex gain, habituation to improve sensitivity to provoking movements and optokinetic exercises to improve visual motion stability. ${ }^{15}{ }^{15}$ An overview of the physiotherapy programme is presented in the online supplemental appendix A. One physical therapist delivers the exercise programme to both groups, including initial regimen and subsequent individualised adjustments.

Group A (UC) will receive instructions from the treating therapist on how to exercise correctly at the first clinic visit. They will then receive once-weekly VRT that lasts at least $12 \mathrm{~min}$ at the clinic and the remaining time homebased exercises for 8 weeks. Video tapes and brochures will be prepared for them to refer to while exercising at home. They are instructed to perform the exercises twice per day with at least 12-minute duration in total and told not to start any new physical activities during the study. Their adherence to the exercise programme will be judged by having them completed a weekly calendar, which will be reviewed by the treating therapist on the once-a-week outpatient clinic. If there is a vacancy in the calendar, the therapist will call the patient to ask the reason for not insisting on coming to the hospital to exercise, such as the difficulty in transportation or the high training intensity, and adjusts accordingly to ensure that the training and follow-up is completed.

Group B (WeChat-VRT) will be provided with adequate supervision of VRT to help them understand the objectives and methods of the project by the physiotherapist during the first clinical visit, in order that they independently manage their own progress, and then all the training will be done at home. Subjects will be offered a series of VRT video demonstrations through WeChat by scanning a unicode, which automatically links to a set of VRT module designed specifically to fit every individual patients' condition (figures 2 and 3 ). The app can also work as a tool recording the time of exercising and uploading videos. The training is assumed to last for 8 weeks and 


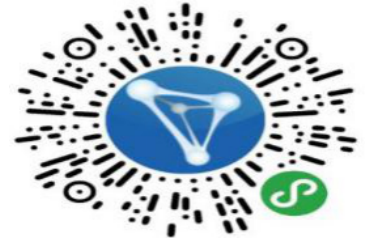

Figure 2 The unicode automatically links to the vestibular rehabilitation therapy module.

the intervention is a combination of home exercise and a weekly message reminder sent by a research coordinator. Subjects are instructed to perform the exercises at home twice per day with at least 12-minute duration in total and told not to start any new physical activities as well.

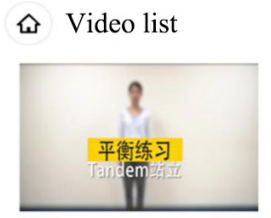

Tandem stand

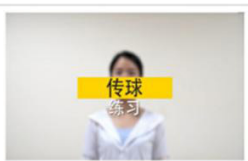

Toss a ball

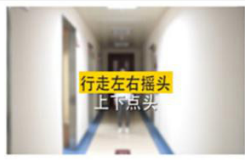

Walk with head shaking

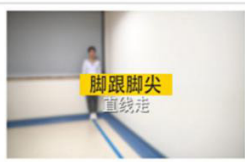

Tandem walk

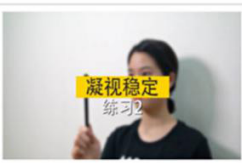

Eye tracking 2

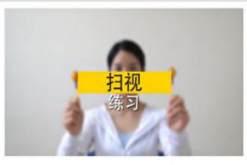

Eye tracking 4

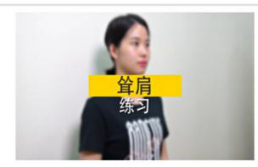

Shrug shoulders

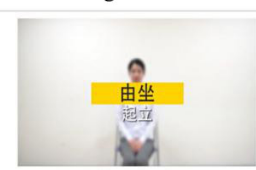

Sit to stand

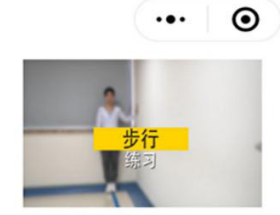

Walk

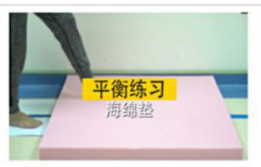

Stand on foam

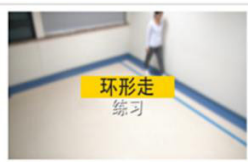

Walk in a circle

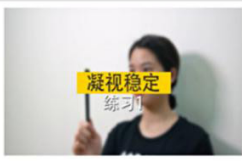

Eye tracking 1

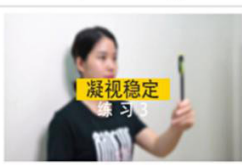

Eye tracking 3

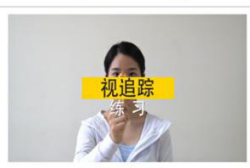

Eye tracking 5

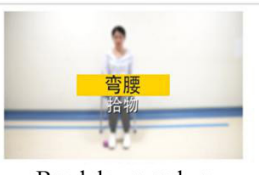

Bend down and up
Figure 3 The playlist of videos that automatically present after scanning the unicode.

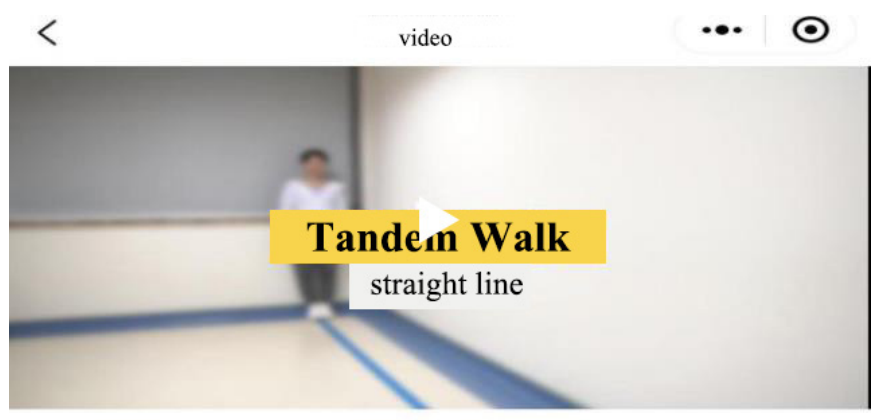

Today record - 0 times

Record your feelings

\section{Record}

Figure 4 WeChat punch the clock page.

Adherence in the WeChat-VRT group will be monitored by having subjects' exercise videos uploaded through WeChat app clock function (figure 4). Only those who have documented their exercise or have uploaded exercising videos will be viewed as being in compliance. If the app does not receive the patient's punch card for 3 consecutive days, it will automatically remind the physiotherapist. She will contact the patient via WeChat or by phone to ask the reason about the unfinished task, such as difficulty in sticking to it, and then make changes or adjustments according to the patient's condition.

For both groups, we will provide a foam as a tool to disturb the somatosensorial system when carrying out exercises. The detailed instruction of using a foam is provided in videos (for the WeChat-VRT group) or brochures (for the UC group). Also, for both groups, we underline the importance of safety before exercise begins and throughout the intervention period. Specifically, two main points are emphasised: first, having someone around during the rehabilitation exercise or do the exercise at a corner with walls or holders being touched when losing balance; second, try to trigger dizziness and/or unsteadiness at some extent, but stop when getting nauseated.

\section{Adverse events}

During VRT, the patients may find that some of the exercises make them uncomfortable, such as dizziness and fatigue. They are encouraged to concentrate and spend extra time on any of the exercises they find difficult. And the patients are instructed to report if any symptoms or 
conditions occur during or after the exercises. There are symptoms that are considered as signs to stop or modify the VRT protocol: vomiting, nausea or muscle soreness; a sharp or prolonged pain sensation in the neck, arms or legs; a sensation of ear fullness, hearing loss or tinnitus; double vision or fainting. The treating physiotherapist will contact the subjects once a week to ask if they had any unexpected symptoms. Any adverse events that may occur during the trial will be recorded within 48 hours and reported to the Adverse Drug Reaction Administration of the Eye and ENT Hospital of Fudan University.

\section{Withdrawal/retention of participants}

Participation in this study is voluntary and participants have the right to decide whether to withdraw at any time. We anticipate some poor adherence and dropouts during the follow-up course. However, we will develop several measures to ensure or improve adherence and to minimise attrition rate. These include giving participants detailed information about the purpose of the study, the treatment options and the importance of follow-up, and the physical benefits they could achieve. Any concerns, such as unexpected symptoms, as well as logistic issues such as travelling to the clinic, parking issues and making appointment with clinical consultant will be evaluated and solved during each telephone consultation. In a limited number of cases, participants may withdraw under certain circumstances, and the reasons for each withdrawal will be truthfully recorded.

\section{Outcome measures}

\section{Primary measure}

Functional capacity is the ultimate objective of rehabilitation. Thus, we chose the change of function as the variable of our primary interest. The Functional Gait Assessment (FGA) will be used as a function assessment in this study. The FGA is consists of 10 items investigating the performance on complex gait tasks (ie, walking with narrow base of support, ambulating backwards, gait with eyes closed, stepping over an obstacle and so on). FGA has demonstrated good internal consistency, with a Cronbach alpha of $0.79 .{ }^{17}$ The highest score is 30. A higher score indicates better performance. The minimal detectable change for FGA is reported to be 6 points in people with vestibular disorders. ${ }^{18}$ Scores $\leq 22 / 30$ are predictable of falls in community-dwelling older adults within 6 months. ${ }^{17}$ In this study, we define the primary outcome as the FGA change from baseline to 8 weeks after allocation.

\section{Secondary outcomes}

1. Balance function, as measured by the Sensory Organization Test (SOT) (Equitest, NeuroCom, Oregon, USA). SOT evaluates the ability of subjects to use vision, vestibular and somatosensory to maintain their balance. ${ }^{19}$ Six increasingly challenging conditions (from SOT1 to SOT6) that disrupt portions of sensory input or visual surroundings are designed to assess balance. Three trials were administered for each condition. An equilibrium score (ES) for each condition is calculated. A composite score (CS) is also calculated as an estimate of overall postural stability.

2. Quality of life, measured by screening version of the Dizziness Handicap Inventory (DHI-S). The DHI-S consists of 10 items. ${ }^{20}$ The DHI-S had been proven to be a valid, reliable and responsive questionnaire that could replace the DHI for daily practice. ${ }^{21}$ At present, since there is no Chinese version of DHI-S in place, our team is currently working on the validation of the DHI-S scale.

3. Emotional well-being including anxiety and depression is measured by Generalised Anxiety Disorder Scale (GAD-7) and Patient Health Questionnaire Depression Scale (PHQ-9). Both questionnaires have been proven as valid instruments.

GAD-7 was developed by Spitzer $e t a l^{22}$ for the screening of generalised anxiety and the assessment of severity of symptom. The scale has seven items designed to understand the patients' mental and emotional changes over the past 2 weeks. The four response categories correspond to $0,1,2$ and 3 points, respectively. The scores ranged from 0 to 21 points, and the sensitivity and specificity were the highest $(89 \%$ and $82 \%$, respectively) at 10 points. GAD-7 can be used not only to monitor GAD but also to assess the severity of the disorder by the scores of 5, 10 and 15 for mild, moderate and severe anxiety disorders, respectively.

PHQ-9 is a self-assessment questionnaire developed by Spitzer $e t a l^{23}$ according to The Diagnostic and Statistical Manual of Mental Disorders, Fourth Edition (DSM-IV) diagnostic criteria. It consists of nine items, which are used to determine how long patients have been suffering from nine problems, including depression, loss of interest and insomnia in the past 2 weeks: $0=$ not at all; $1=$ a few days; $2=$ more than half of the days; $3=$ almost every day. The total score of the scale was 27 , with $0 \sim 4,5 \sim 9,10 \sim 14,15 \sim 27$ representing zero, mild, moderate and severe depression, respectively.

4. Activity and participation level will be assessed by Vestibular Activities and Participation measure (VAP), which was developed based on the International Classification of Functioning, Disability and Health (ICF) from the WHO. The Chinese version of VAP had been validated in patients with vestibular disorder. ${ }^{24}$ Patients are instructed to choose 'no difficulty', 'mild difficulty', 'moderate difficulty' or 'severe difficulty' to describe how difficult it is to perform the related activity recently due to dizziness or difficulty in balance. According to the cut-off score, the practitioners can detect clinically significant activity and participation impairment in the individuals with vestibular disorders.

\section{Timepoints of outcome measurements}

Demographic and clinical data collection will include age, sex, education, employment, marital status, coexisting systemic diseases, date of onset, duration of symptoms from onset to treatment and affected ear. Participants are 


\begin{tabular}{|c|c|c|c|c|c|}
\hline Task & Screening & Baseline & 8th week & 12th week & 24th week \\
\hline Eligibility screening and informed consent form & $\checkmark$ & - & - & - & - \\
\hline Physical examination & $\checkmark$ & - & - & - & - \\
\hline Allocation & $\checkmark$ & - & - & - & - \\
\hline Assessments of outcome variables & & $\checkmark$ & $\checkmark$ & $\checkmark$ & $\checkmark$ \\
\hline
\end{tabular}

VRT, vestibular rehabilitation therapy.

required to return to the clinic at the 8 th, 12th and 24th week post-allocation. The timeline is presented in table 1 .

\section{Sample size calculation}

The study sample size justification is based on the primary outcome-FGA measure. The minimum detectable change (MDC) for the FGA is reported to be 6 points, with an SD of 5.5, as reported previously. ${ }^{13}$ In this study, we assume an FGA difference of 6 will be considered clinically meaningful. Thus, to detect an MDC of 6 for the FGA with $80 \%$ power (alpha level of 0.05 , two-tailed test), we will require 21 subjects per group. Allowing for a $15 \%$ dropout rate, the sample size inflates to 25 subjects for each group (50 in total).

\section{Data analysis}

The main analysis of change in the primary and secondary outcomes (FGA score, SOT composite, GAD-7 and PHQ-9 scores) in baseline and three follow-ups will use generalised estimation equation for the two groups. Models will be adjusted for age, sex and other baseline potential confounders. The information of medication taken during the study period will be collected by the blinded assessor and incorporated as a confounder. Adherence rate and dropout rate between the two groups will be compared by using the $\chi^{2}$ test. Statistical analysis will be carried out by a statistician (HZ) outside the study team, who is blinded to group allocations and the study hypotheses. To achieve blinding, all subjects will be anonymous, and study groups will be coded as A, B. Since the dropout/attrition rate is anticipated, the primary analysis of the outcomes will be on the conservative intentionto-treat (ITT) basis, with participants analysed based on their initial allocation group; meanwhile, a per-protocol (PP) analysis will also be performed as a secondary analysis (defined as completing at least one follow-up). We will check the structure and pattern of missing data and, if appropriate, multiple imputation approaches will be used in both the ITT and the PP analyses.

\section{Study status and recruitment}

This is protocol V.1.1, which was updated on 25 March 2020. The planned date of first enrolment is 1 June 2021. The estimated time required for recruitment is 12 months. The total duration of this study is expected to be
24 months, including statistical analysis and drafting of the study results.

\section{Data management}

The team will set up a data management group consisting of a group leader, a statistician, a methodologist, an experienced physiotherapist specialised in vestibular rehabilitation and a patient representative. All individuals are independent of the study sponsor and have no conflict of interests. Once the experiment begins, the data management team will meet monthly to manage and monitor the daily operation of the experiment, review existing data, check the integrity of the database and complete the interim analysis. The sponsor will pay for their time and efforts.

During the trial, all the data at baseline and the three follow-up visits will be recorded in an electronic case specifically designed. Electronic data will be stored on a public platform at http://www.medresman.org, which is sponsored by the China Clinical Trial Registry. The database uses standard techniques to provide security. Encrypted passwords are needed when the researchers access the database. Individual participant data (IPD) will be made public 6 months after the completion of the study via the IPD sharing platform.

\section{Patient and public involvement}

Patients have been involved in the design of this study. We have interviewed three patients with chronic vestibular syndrome about their views on the intensity of training, the frequency of interaction and guidance. Their views have been incorporated into our revised protocol. Patients and the public will be informed of the results through peer-reviewed journals or academic conferences.

\section{ETHICS AND DISSEMINATION}

This study was approved by the Institutional Review Board of Fudan University Eye Ear Nose and Throat Hospital (reference number 2017047/1). The informed consent and confidentiality principles will be followed throughout the experiment. We plan to publish the study findings in peer-reviewed academic journals. We also intend to present this study at local, national and international 
conferences where possible. Furthermore, we will draft a summary of the study results to be posted on the website of eye and ENT hospital that can be accessed by all trial participants as well as relevant interest groups.

Contributors All authors have made an intellectual contribution to this protocol. SX and HZ held full responsibility for this manuscript. PW, YW, YZ and CW conceived the design, developed the protocol and wrote the first draft of this manuscript. SX and $\mathrm{HZ}$ revised and approved the final version of the study protocol and the final manuscript. SX and $\mathrm{HZ}$ contributed equally.

Funding This study was supported by the 2019 Clinical research project of Shanghai Health and Family Planning Commission (Grant No. 20190046) and Science and Technology Commission of Shanghai Municipality ((STCSM), Grant No. 184119551900).

Competing interests None declared.

Patient consent for publication Obtained.

Provenance and peer review Not commissioned; externally peer reviewed.

Supplemental material This content has been supplied by the author(s). It has not been vetted by BMJ Publishing Group Limited (BMJ) and may not have been peer-reviewed. Any opinions or recommendations discussed are solely those of the author(s) and are not endorsed by BMJ. BMJ disclaims all liability and responsibility arising from any reliance placed on the content. Where the content includes any translated material, BMJ does not warrant the accuracy and reliability of the translations (including but not limited to local regulations, clinical guidelines, terminology, drug names and drug dosages), and is not responsible for any error and/or omissions arising from translation and adaptation or otherwise.

Open access This is an open access article distributed in accordance with the Creative Commons Attribution Non Commercial (CC BY-NC 4.0) license, which permits others to distribute, remix, adapt, build upon this work non-commercially, and license their derivative works on different terms, provided the original work is properly cited, appropriate credit is given, any changes made indicated, and the use is non-commercial. See: http://creativecommons.org/licenses/by-nc/4.0/.

ORCID iD

Peixia Wu http://orcid.org/0000-0003-4946-4140

\section{REFERENCES}

1 Agrawal Y, Van de Berg R, Wuyts F, et al. Presbyvestibulopathy: diagnostic criteria consensus document of the classification Committee of the Bárány Society. J Vestib Res 2019;29:161-70.

2 Strupp M, Kim J-S, Murofushi T, et al. Bilateral vestibulopathy: diagnostic criteria consensus document of the classification Committee of the Bárány Society. J Vestib Res 2017;27:177-89.

3 Odman M, Maire R. Chronic subjective dizziness. Acta Otolaryngol 2008;128:1085-8.

4 Yardley L, Owen N, Nazareth I, et al. Prevalence and presentation of dizziness in a general practice community sample of working age people. Br J Gen Pract 1998;48:1131-5.
5 Hannaford PC, Simpson JA, Bisset AF, et al. The prevalence of ear, nose and throat problems in the community: results from a national cross-sectional postal survey in Scotland. Fam Pract 2005;22:227-33.

6 Bisdorff A, Bosser G, Gueguen R, et al. The epidemiology of vertigo, dizziness, and unsteadiness and its links to co-morbidities. Front Neurol 2013;4:29.

7 Kroenke K, Price RK. Symptoms in the community. Prevalence, classification, and psychiatric comorbidity. Arch Intern Med 1993;153:2474-80.

8 Bronstein AM, Lempert T. Management of the patient with chronic dizziness. Restor Neurol Neurosci 2010;28:83-90.

9 Sloane PD, Coeytaux RR, Beck RS, et al. Dizziness: state of the science. Ann Intern Med 2001;134:823-32.

10 Hall CD, Herdman SJ, Whitney SL, et al. Vestibular rehabilitation for peripheral vestibular hypofunction: an evidence-based clinical practice guideline: from the American physical therapy association Neurology section. J Neurol Phys Ther 2016;40:124-55.

11 van Vugt VA, van der Horst HE, Payne RA, et al. Chronic vertigo: treat with exercise, not drugs. BMJ 2017;358:j3727.

12 Zhang X, Xiao H, Chen Y. Evaluation of a WeChat-based life review programme for cancer patients: a quasi-experimental study. J Adv Nurs 2019;75:1563-74.

13 Jiang Y, Liu F, Guo J, et al. Evaluating an intervention program using WeChat for patients with chronic obstructive pulmonary disease: randomized controlled trial. J Med Internet Res 2020;22:e17098.

14 Chun L M. Effect of WeChat combined with health belief model on home based exercise after knee surgery. International Journal of Nursing(in Chinese ) 2020;39:3440-1.

15 Whitney SL, Alghwiri AA, Alghadir A. chapter editor. "An overview of vestibular rehabilitation". In: Handbook of clinical neurology, 2016: 187.

16 Herdman SJ. Vestibular rehabilitation. Curr Opin Neurol 2013;26:96-101.

17 Wrisley DM, Marchetti GF, Kuharsky DK, et al. Reliability, internal consistency, and validity of data obtained with the functional gait assessment. Phys Ther 2004;84:906-18.

18 Marchetti GF, Lin C-C, Alghadir A, et al. Responsiveness and minimal detectable change of the dynamic gait index and functional gait index in persons with balance and vestibular disorders. $J$ Neurol Phys Ther 2014;38:119-24.

19 Nashner LM. Computerized dynamic posturography. In: Jacobson GP, Shepard NT, eds. Balance function assessment and management. California: Plural Publishing Inc, 2014: 451-79.

20 Jacobson GP, Calder JH. A screening version of the dizziness handicap inventory (DHI-S). Am J Otol 1998;19:804-8.

21 van Vugt VA, de Vet HCW, van der Wouden JC, et al. The 25-item dizziness handicap inventory was shortened for use in general practice by 60 percent. J Clin Epidemiol 2020;126:56-64.

22 Spitzer RL, Kroenke K, Williams JBW, et al. A brief measure for assessing generalized anxiety disorder: the GAD-7. Arch Intern Med 2006;166:1092-7.

23 Spitzer RL, Kroenke K, Williams JB. Validation and utility of a selfreport version of PRIME-MD: the PHQ primary care study. primary care evaluation of mental disorders. patient health questionnaire. JAMA 1999;282:1737-44.

24 Wu P, Yang M, Hu Y, et al. Cross-cultural adaptation and validation of the Chinese version of the vestibular activities and participation measure. J Vestib Res 2019;29:171-9. 